\title{
About waveguide modeling (transmissions of ultrasonic technological machines)
}

\author{
Vitaly Krupenin and Vladimir Astashev \\ Mechanical Engineering Research Institute of the Russian Academy of Sciences, Moscow, Russian Federation
}

\begin{abstract}
The modeling of energy transfer systems in ultrasonic technological machines is considered. As such systems rod waveguides are usually used. These are, as a rule, distributed systems of variable crosssection, which must transmit energy from an electromechanical vibration exciter to a working body. Sometimes additional equipment is attached to the waveguide (core system), which should be modeled using lumped-parameter subsystems. This article discusses the problems related to the modeling and calculation of the so-called ultrasonic concentrators - waveguides that have a heterogeneous shape and are made to ensure optimal energy delivery to the working body (instrument). Examples of calculations are given that consider the influence of a nonlinear elastic-dissipative load, determined by the nature of the technological process. The analysis of real models is described.
\end{abstract}

\section{Introduction}

Ultrasonic technologies make it possible to organize a universal way of highly effective effects on workpieces and environments. Three main components define ultrasonic machines: "electromechanical vibration exciter - waveguide (concentrator) - technological process". Thus, waveguides define the transmission systems of ultrasonic machines. This is, as a rule, a rod system with distributed parameters, designed to transfer the energy of the exciter to the working part of the machine.

This paper discusses the problems of modeling and calculating the so-called ultrasonic concentrators waveguides, which have a non-uniform shape and are made to ensure optimal energy delivery to the instrument.

The theory of ultrasonic concentrators, suggesting their perfect matching at idle, is developed in [1-5]. Below will be considered models of rod concentrators with internal energy loss.

The methods for calculating waveguides for ultrasonic machines under the assumption of linearity of rod systems are based on the methods of dynamic compliance, which are determined by solving the corresponding equations of motion in partial derivatives. When considering in the model of the workflow, which consists, in particular, in the interaction of the tool with the processed medium or workpieces, the equations of motion become strongly nonlinear and the methods for their analysis change. An efficient and fairly easy to interpret way to study the models under consideration is provided by frequency methods based on the ideas of equivalent linearization.

\section{Source models}

Consider the work of the ultrasonic technological machine. The cross-section $x=0$ of the concentrator 1 (Fig.1) is connected with the exciter oscillations 2, and in the section $x=l$ the tool 3 is fixed, which interacts with the workpiece or medium 4 .

The oscillations of the cross section $x$ of the concentrator will be described by the function $u_{x}(t)=a_{x} \exp i\left(\omega t-\varphi_{x}\right)$, where $a_{x}, \omega, \varphi_{x}-$ amplitude, frequency and initial phase.

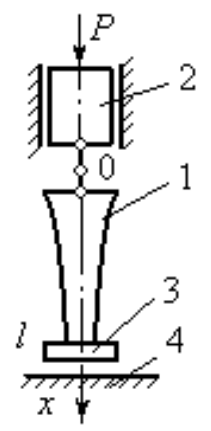

Fig. 1. The scheme of the ultrasonic process: 1- concentrator; 2- exciter oscillations; 3-tool; 4- processed medium.

In technological ultrasonic machines and devices, the load on the oscillatory system from the side of the processed workpieces and media is, as a rule, non-linear. The corresponding reaction force can be represented as

\footnotetext{
*Corresponding author: krupeninster@gmail.com
} 
follows: $f\left(u_{l}\right)=\left[k\left(a_{l}\right)+j \omega \beta\left(a_{l}\right)\right] u_{l}$. Here and below, nonlinear forces are written using techniques equivalent linearization [4, 5]. The nonlinearity of the load is manifested in the fact that its elastic and dissipative components are dependent on the amplitude of oscillations of the tool. This dependency was repeatedly observed experimentally and was described in detail for a long time [6].

Mutual influence of the concentrator and the causative agent of oscillations will be assessed by the force acting in the place of their joint $f_{0}(t)=F_{0}$ expi $i t$.

The motion of the concentrator will be determined using the magnitude of the dynamic compliance $L_{s}(x, i \omega)$ that relates the displacement of $u_{x}$ cross sections $x$ with reactions $f\left(u_{l}\right), f_{0}(t)$ and the inertial force $-M \omega^{2} u_{l}$ from the attached tool with a mass $M$ acting in cross sections $s=0, l$ :

$$
u_{x}(t)=L_{0 x}(i \omega) F_{0} \exp i \omega t-L_{0 x}(i \omega)\left[g\left(a_{l}\right)-M \omega^{2}\right] u_{l},
$$

and $g\left(a_{l}\right)=k\left(a_{l}\right)+\beta\left(a_{l}\right)$. After a series of transformations, we find the formulas for the amplitude and phase of moving the cross-section of the concentrator at $x=l$ :

$$
\begin{aligned}
& a_{l}=F_{0}\left|W\left(a_{l} i \omega\right)\right|^{-1}=F_{0}\left[(\operatorname{Re} W)^{2}+(\operatorname{Im} W)^{2}\right]^{-0,5} \\
& \cos \varphi_{l}=a_{l} F_{0}^{-1} \operatorname{Re} W\left(a_{l}, i \omega\right) ; \sin \varphi_{l}=a_{l} F_{0}^{-1} \operatorname{Im} W\left(a_{l}, i \omega\right) .
\end{aligned}
$$

We introduce the designation of dynamic stiffness:

$W\left(a_{l}, i \omega\right)=\left\{1+L_{l}(l, i \omega)\left[k\left(a_{l}\right)-M \omega^{2}+i \omega \beta\left(a_{l}\right)\right]\right\} L_{0}^{-1}(l, i \omega)$. Let $W_{s x}(i \omega)=U_{s x}(\omega)+i V_{s x}(\omega)$.

It is assumed that the level of damping is small, i.e. small quantities $V_{s x}(\omega), \beta\left(a_{l}\right)$. Equation (2) determines the amplitude-frequency characteristic of the concentrator when a force acts at the end of $x=0$. The maximum amplitude of the instrument is achieved under the condition $\operatorname{Re} W\left(a_{l}, i \omega\right)=0$, which can be brought to the following form: $U_{l l}(\omega)+k\left(a_{l}\right)-M \omega^{2}=0$.

In this case, of course, any given amplitude $a_{l}$ is provided by some minimum force $F_{0}$. Consequently, the last relation turns out to be the condition for the best matching of the vibration exciter with the concentrator. We emphasize that in this case the concentrator carries a tool with a mass $\mathrm{M}$ and operates on a non-linear technological load.

Considering the obtained equalities, we find

$$
K=\frac{a_{l}}{a_{0}}=\left|\frac{U_{00}(\omega)}{U_{0 l}(\omega)}\right| .
$$

By analogy with $[1,2,5]$, the value $K=a_{l} / a_{0}$ characterizing the degree of transformation of the level of oscillations transmitted from the exciter to the tool will be called the gain coefficient of the concentrator. Note that the expression (3) in its form coincides with the previously known equality $[4,5]$, which determines the gain factor of the unloaded concentrator. For a given amplitude value $a_{0}$ of the exciter of the oscillations, the relation (3) immediately determines the amplitude of the working end of the concentrator, which satisfies the given condition of the best matching under load.

\section{Attaching a concentrator}

It should be noted that the attachment of the concentrator leads to the loading of the pathogen. Let the pathogen is not loaded by the concentrator and oscillates in accordance with the law of motion. $u_{0}^{*}(t)=$ $=a_{0}^{*} \exp i(\omega t-\varphi)$.

At the point of connection of the concentrator on the vibration exciter force $-f_{0}(t)$. His movement under load will be described by the equation $u_{0}(t)=u_{0}^{*}(t)-f_{0}(t) W_{0}^{-1}(i \omega), \quad$ where $\quad W_{0}(i \omega)=$ $=U_{0}(\omega)+i V_{0}(\omega)$ - dynamic stiffness of the exciter at the point of attachment of the concentrator.

Using the derived ratios, the magnitude of the reaction can be represented as $f_{0}(t)=i u_{0}(t) K^{2}\left[V_{l l}(\omega)+\omega \beta\left(a_{l}\right)\right]$.

In the books $[4,5]$, it was shown that in ultrasonic technological installations, resonant electroacoustic transducers are usually used as exciters. The condition of their resonant tuning is $U_{n}(\omega)=0$. Now for the loaded transducer we get:

$$
u_{0}(t)=u_{0}^{*}(t)\left[1+V_{l l}(\omega)+\omega \beta\left(a_{l}\right) V_{n}^{-1}(\omega) K^{2}\right]^{-1} .
$$

It follows that the connection of a consistent concentrator does not change the resonant tuning of the converter. At the same time, the change in the level of its oscillations is determined by the ratio of the dissipative parameters of the converter, the concentrator and the load. From the relation (3) considering the formula (4) we find the amplitude of oscillations of the tool

$$
a_{l}=a_{0}^{*} K\left[1+V_{l l}(\omega)+\omega \beta\left(a_{l}\right) V_{n}^{-1}(\omega) K^{2}\right]^{-1} .
$$

\section{Exponential concentrator}

Let's consider, skipping some transformations, a situation arising from the ultrasonic processing of fragile materials. In $[4,5]$ it is shown that the processing occurs as a result of high-frequency vibro-impact interactions of the tool with the workpiece during their compression by the static force $P$. There, on the basis of the rheological model of the cutting process, expressions for the elastic and dissipative components of the load were obtained:

$$
k\left(a_{l}\right)=\frac{D}{\pi a_{l}} \sin \frac{2 \pi P}{D} ; \beta\left(a_{l}\right)=\frac{2 D}{\pi \omega a_{l}} \sin ^{2} \frac{\pi P}{D},
$$


where $\mathrm{D}$ is a value proportional to the area of the tool and depends on the material of the product, as well as the type of abrasive slurry.

After substituting expressions (6) into the formulas of the previous section, we obtain, after transformations, a system of equations, the solution of which determines the parameters and amplitude of oscillations of the concentrator matched under the load:

$$
\begin{aligned}
& a_{l}=\frac{2 P}{M \omega^{2}-U_{l l}(\omega)} \\
& a_{l}=a_{0}^{*} K\left[1-K \frac{\pi P^{2}}{D V_{n}(\omega)}\right]\left[1+K^{2} \frac{V_{l l}(\omega)}{V_{n}(\omega)}\right]^{-1}
\end{aligned}
$$

Below are the results of the calculation of one of the most common type of ultrasonic concentrator. The concentrator 1 (Fig.1) at its end connected to the output of the oscillation exciter 2.

Dynamic compliance of concentrators of various types are defined in $[4,5]$ by solving the equations of longitudinal vibrations of rods of variable cross section.

Consider an exponential concentrator, the area $S_{x}$ of the cross-section $\mathrm{x}$ of which varies according to the law $S_{x}=S_{0} \exp (2 \gamma x / l), \gamma=1 / 2 \ln \left(S_{l} / S_{0}\right)$.

Expression for dynamic compliances of an exponential waveguide were obtained in $[4,5]$ and for the corresponding operator $L_{l x}(i \omega)$ has the form

$$
L_{l x}(i \omega)=\frac{\gamma \operatorname{sh} \lambda x-\lambda l \operatorname{ch} \lambda x}{\omega^{2} \rho S_{0} l \operatorname{sh} \lambda} e^{\gamma\left(1+\frac{x}{l}\right)} .
$$

где $\lambda=\frac{\omega}{c}\left(i \frac{c^{2}}{c_{\mathrm{Tм}}^{2}}+\frac{\psi}{2 \pi}\right)^{1 / 2} ; c_{\phi}=\frac{c}{\sqrt{1-(\gamma c / l \omega)^{2}}}$

- phase velocity of sound in an exponential waveguide; $\quad c=\sqrt{E / \rho}$ - sound velocity in the concentrator material; $E, \rho$ - elastic modulus and density of the concentrator material.

Suppose that the absorption coefficient [7] $\psi$, which describes the energy loss in the material of the concentrator, is small. We will find the expression for the components of the dynamic rigidity of the exponential concentrator and then we obtain two equations that determine the desired solution. Let $\xi=\omega l c_{\phi}^{-1}$. Considering formula (9), by conducting a series of transformations, relation (3) for the gain of a loaded concentrator can be converted to the following form

$$
K=\frac{a_{l}}{a_{0}}=\left|\frac{\gamma \cos \xi+\xi \sin \xi}{\xi}\right| \sqrt{\frac{S_{0}}{S_{l}}}
$$

After calculations, you can also get:

$$
a_{l}=\frac{2 P}{\omega w_{l}}\left(\mu+\frac{\sqrt{1+(\gamma / \xi)^{2}}}{\cos \xi+(\gamma / \xi) \sin \xi}\right)^{-1}
$$

where $\mu=M \omega / w_{l} ; w_{l}=S_{l} \sqrt{E \rho}$ - at the output end of the concentrator.

The joint solution of equations (10) and (11) determines the desired values of $\xi$ and $a_{l}$ for an exponential concentrator that is matched under load. Here the amplitude value at the input end of the concentrator is set. This solution is convenient to perform numerically. The length of the concentrator is determined by the formula $l=c \omega\left(\xi^{2}+\gamma^{2}\right)^{0,5}$. That in the absence of load $(\xi=\pi n)$ coincides with that obtained in [3-5].

Fig. 2 shows the dependences of the length $l$ of the concentrator, matched under load, on the compressive force $P$ for different values of the mass of the tool $M$. Here $l_{0}$ - the length of the free unloaded concentrator, which is determined by $\xi=\pi n$. In the calculations it was assumed: the oscillation frequency $f=\omega / 2 \pi=20 \mathrm{kHz}$;

$K=4 ; w_{l}=3,14 \cdot 10^{3} \mathrm{~N} \cdot \mathrm{s} / \mathrm{m} ; \psi=0,01 ; D=5 \cdot 10^{4} \mathrm{~N}$.

At Fig. 2 : $1-\mu=0 ; 2-\mu=0,2 ; 3-\mu=0,4 ; 4-\mu=0,6$; $5-\mu=0,8 ; 6-\mu=1$.

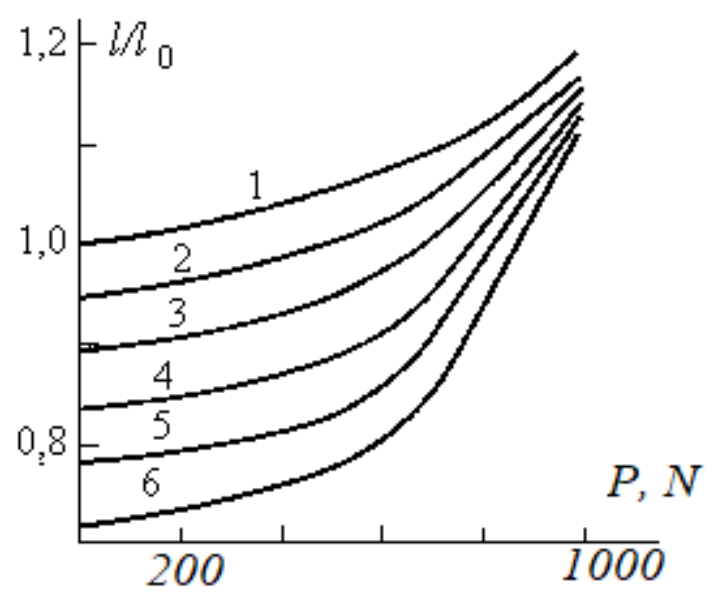

Fig. 2. Concentrator length.

On this research problem cannot be completed. This is due to the fact that the connection of the concentrator affects the amplitude of the electroacoustic transducer. From the formula (8) it can be seen that the amplitude of oscillations at the output end of the concentrator depends on the ratio of dissipative losses in the converter and the concentrator.

Let, for definiteness, the transducer is a homogeneous rod of length $l_{n}$ with a cross section $S_{n}$. Its dynamic stiffness tuning in resonance to the frequency of the first form of natural oscillations is given by the formula [4]: $V_{n}=0,25 \psi_{n} \omega w_{n}$, where $w_{n}=S_{g} \sqrt{E_{n} \rho_{n}}$ wave resistance of the converter at the concentrator's connection point; $E_{n}, \rho_{n}, \psi_{n}$ - elastic modulus, density and absorption coefficient of the converter material [7]. Joining even an unloaded $(P=0, M=0)$ concentrator to a resonant vibration exciter changes its amplitude, which is found from the above formulas, so that 


$$
a_{l}^{*}=a_{0}^{*} K\left[1+\frac{\psi w_{l} K^{2}\left(1+\gamma^{2} / n^{2} \pi^{2}\right)}{\psi_{n} w_{n}}\right]^{-1} .
$$

From the last expression it is seen that the amplitude of oscillations of a concentrator with internal losses decreases in comparison with the ideal one. This decrease is the more significant, the greater the magnitude of these losses. The change in amplitude at the output of the hub depends on its gain coefficient $K$.

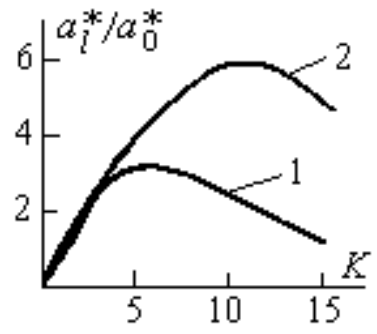

Fig. 3. The oscillation amplitude of the end of the concentrator

In Fig.3, formula (12) is used to build the dependence of the amplitude of oscillations of the free end of the exponential concentrator on the gain coefficient $\mathrm{K}$ (curve). Here $\psi_{n} w_{a} / \psi w_{l}=65$. This shows that there is a gain coefficient $\mathrm{K}$ value at which the amplitude of oscillation of the output end of the concentrator is maximum.

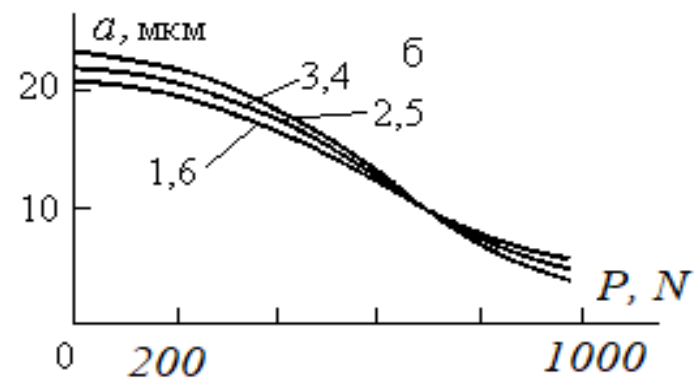

Fig. 4. The dependence of the amplitude on the compressive force.

In Fig.4, the dependences of the amplitude of oscillations of an exponential concentrator matched under load on the compressive force for various tool weights are plotted. The graphs are plotted with the same parameters as Fig.2. It was selected $\omega_{n}=4,084 \cdot 10^{4} \mathrm{~N} \cdot \mathrm{s} / \mathrm{m}$; $\psi_{n}=0,05$.

Research models of other types of concentrators is performed similarly. For example, curve 2 in Fig. 3 corresponds to a step type concentrator consisting of two cylindrical bars $[4,5]$. The corresponding calculation is not given here.

\section{Conclusions}

The analysis of concentrator models in ultrasonic technological machines and devices is reduced to the study of the features of longitudinal oscillations of rods with variable cross sections. Such rods function in the presence of an elastic-dissipative load, as well as several additional factors. At the same time, it is necessary to consider the dissipative properties of the material of the concentrator itself. As a result, the studied models turn out to be essentially nonlinear. Their research and description can be performed using frequency methods based on ideas of equivalent linearization. These methods allow us to give an interpretation of the main dynamic effects, manifested in concentrators of different types.

The paper was supported by the Russian Science Foundation (project \# 18-08-00168).

\section{References}

1. L. Merkulov Acustical journal 3, 3 (1957)

2. L. Merkulov, A. Kharitonov Acustical journal 5, 2 (1959)

3. I. Teumin Ultrasonic oscillating systems (Machgiz, Moscow, 1959)

4. V. Astashev, V. Babitsky Ultrasonic Proocesses and Machines (Springer, 2007)

5. V. Astashev, V. Krupenin Non- linear Dynamics of Ultrasonic Technological Processes (MGUP im Ivana Fedorova, Moscow, 2016)

6. L. Rozenberg, V. Kazantsev Machine-tools and tools 5 (1959)

7. E. Sorokin To the theory of internal friction in the oscillation of elastic systems (Gosstroiizdat, Moscow, 1966) 